Documentation et bibliothèques

DOCUMENTATION BIBLIOTHËQUES

\title{
Transition du système informatisé CATSS de l'École Polytechnique à celui de GLIS : aspects organisationnels et aspects humains
}

\section{André Maltais et Denise Mauffette}

Volume 32, numéro 3, juillet-septembre 1986

URI : https://id.erudit.org/iderudit/1052674ar

DOI : https://doi.org/10.7202/1052674ar

Aller au sommaire du numéro

Éditeur(s)

Association pour l'avancement des sciences et des techniques de la documentation (ASTED)

ISSN

0315-2340 (imprimé)

2291-8949 (numérique)

Découvrir la revue

Citer cet article

Maltais, A. \& Mauffette, D. (1986). Transition du système informatisé CATSS de l'École Polytechnique à celui de GLIS : aspects organisationnels et aspects humains. Documentation et bibliothèques, 32(3), 87-92.

https://doi.org/10.7202/1052674ar

Tous droits réservés (C) Association pour l'avancement des sciences et des techniques de la documentation (ASTED), 1986
Ce document est protégé par la loi sur le droit d'auteur. L'utilisation des services d'Érudit (y compris la reproduction) est assujettie à sa politique d'utilisation que vous pouvez consulter en ligne.

https://apropos.erudit.org/fr/usagers/politique-dutilisation/ 


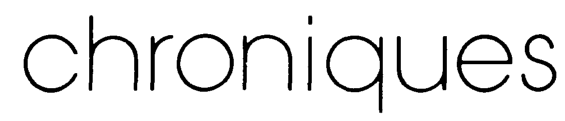

\section{Transition du système informatisé CATSS de l'École Polytechnique à celui de GLIS: aspects organisationnels et aspects humains}

En 1977. la bibliothèque de l'École Polytechnique de Montréal informatisait son service de catalogage. C'était à UTLAS que nous faisions I'honneur (disons-le franchement) de devenir leur client. Pendant neuf ans, nous avons créé notre fichier comprenant 50000 titres de publications gouvernementales et 45000 autres périodiques ou monographies. Notre fichier autorité comprenait 15000 vedettes d'identification incluant des noms de collectivités, de conférences et des titres de collections dont nous contrôlions la forme.

En 1984, le besoin d'informatiser les autres services de la bibliothèque se fit sentir. UTLAS n'étant pas en mesure de nous offrir un système intégré complet, il nous a fallu considérer la possibilité de changer de système. C'est finalement le logiciel GEAC Library Information System (GLIS) de GEAC qui a été choisi.

Pour le personnel des services techniques et plus particulièrement celui du catalogage, cela signifiait de vivre une expérience que peu de gens ont vécue jusqu'à maintenant: passer d'un système informatisé à un autre. Nous verrons comment le personnel du service de catalogage à vécu cette transition, comment il a absorbé le surplus de travail inévitable qui en découlait et comment, avec très peu de ressources humaines additionnelles, le très court calendrier d'implantation fut respecté.

\section{Organisation du service de catalogage}

Pendant le règne d'UTLAS et encore aujourd'hui, le service de catalogage de notre bibliothèque cohabite avec celui des acquisitions pour former les services techniques. Le responsable du catalogage travaille sous l'autorité du responsable des services techniques. Le personnel est composé de trois unités ou équipes de travail formées chacune d'un bibliothécaire et de deux techniciennes en documentation. La première de ces unités assume le catalogage des monographies, la seconde celui des périodiques et la troisième, celui des publications gouvernementales et des organismes de recherche. Deux commis sont de plus affectés à la préparation matérielle des documents traités.
À l'automne 1984, la responsable du service de catalogage est promue chargée de projet pour toute la période d'implantation du nouveau système. En plus de se familiariser avec tous les dossiers d'UTLAS, son successeur doit assimiler la nouvelle version de CATSS, assurer l'entraînement de tout le personnel et former son successeur dans l'équipe des monographies. Cette personne est embauchée en février 1985 au beau milieu des premiers essais... laborieux du système CATSS II et des préparatifs pour la conversion à GLIS.

\section{Conversion des notices}

À l'été 1985, la seconde étape de l'implantation du nouveau système consista à vérifier les 6000 notices catalographiques en format MARC qui venaient d'être converties de UTLAS à GLIS. Elles

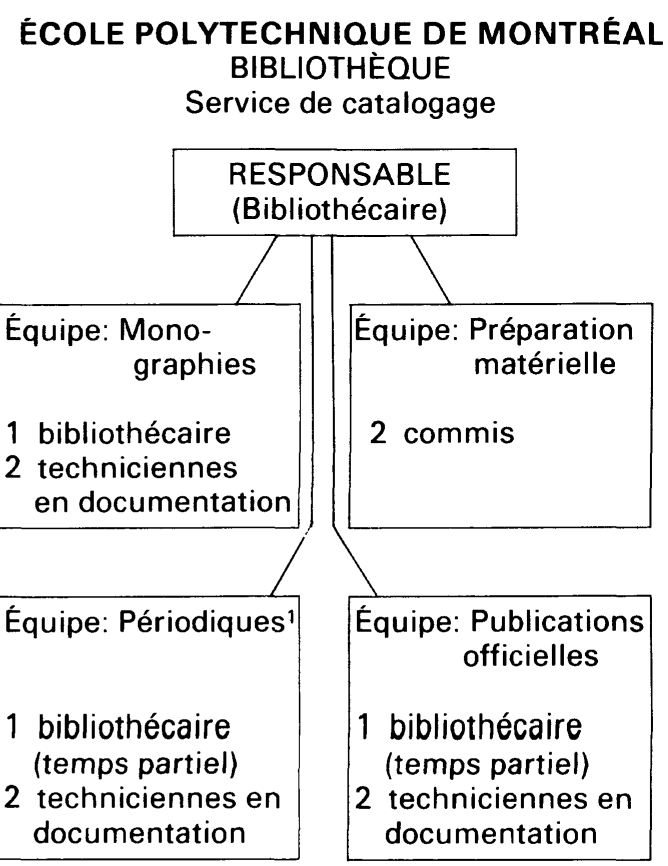

1. Dans cette équipe, $80 \%$ du catalogage est rétrospectif. 
étaient regroupées sur une bande magnétique. La figure 1 illustre une partie spécifications de conversion (une quarantaine).

Le travail a été également réparti entre les quatre bibliothécaires et les six techniciennes en documentation, ce qui représentait 600 notices par personne. En plus de vérifier l'exactitude de la conversion, il fallait noter ce qui n'avait pas fonctionné afin d'en informer la chargée de projet.

Tout le personnel a été convié à trois journées de formation au fonctionnement des terminaux GEAC et à la structure du module de catalogage GLIS. Si nous ne pouvions espérer des ressources supplémentaires en personnel, les travaux devaient être terminés le 16 août, trois semaines après le début!

\section{Opération codes zébrés}

Le 17 juin débuta une opération très importante de pose de codes zébrés sur chacun de 90000 documents prêtables de la bibliothèque en prévision du fonctionnement du module de prêt GEAC.

Deux commis ont été embauchées pour une période de cinq mois et ont effectué de $85 \%$ à $90 \%$ du travail de zébrage. Le reste a été partagé entre deux commis à la préparation matérielle et tout le personnel de la bibliothèque. En effet, à la demande du directeur les quarante-cinq employés de la bibliothèque ont consacré une journée de leur temps à la pose des étiquettes sur les volumes.

Le service de catalogage a supervisé cette opération. Deux techniciennes en documentation ont été affectées à la solution des cas-problèmes et, devant l'amoncellement des cas à traiter, une troisième personne à dû joindre l'équipe de travail. II faudrait peut-être expliquer ici la raison des nombreux cas-problèmes.

Nous ne voulions et ne pouvions pas entrer des codes zébrés dans GLIS en descendant physiquement toute la collection aux services techniques, situés au $3 e$ étage. Comme la collection zébrée est au $4 \mathrm{e}$ étage, c'est donc deux fois le même code zébré qui devait être posé pour chaque unité documentaire prêtable de la bibliothèque. Le premier exemplaire était posé sur le document et le second sur la fiche topographique d'UTLAS correspondant à ce document. Ainsi, plus tard, on entrerait dans GLIS le code zébré de chaque document à partir des fiches topographiques.

Mais le risque d'erreur était plus élevé parce que les commis devaient poser les bons codes zébrés aux endroits appropriés tout en identifiant clairement chacun d'eux. L'exemple suivant tiré du procédurier préparé par le responsable du trai- tement de la documentation, illustre la fragilité de l'opération et, par conséquent les nombreuses erreurs possibles. Rappelons qu'une fiche topo pouvait contenir jusqu'à 83 codes zébrés identifiés de façons très variées: «1945»; «1946, ex. 1»; «1946, ptie 1, ex. 2»; «vol. 3, fasc. A»; «1984/1985, ptie B-5, suppl. », etc.

\section{Le code zébré}

1. Les codes zébrés sont toujours fournis par paires (deux exemplaires parfaitement identiques disposés l'un sur l'autre).

Le deuxième exemplaire porte toujours la mention «DUPL» dans le coin supérieur droit.

\section{Exemples:}

1 er exemplaire

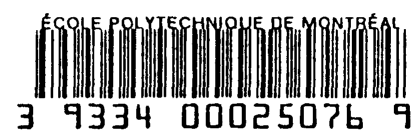

2e exemplaire

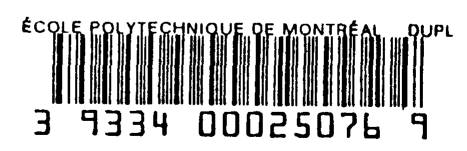

2. Quand on trouve une fiche et un livre qui ont exactement la même cote, on pose le premier exemplaire du code zébré sur le livre:

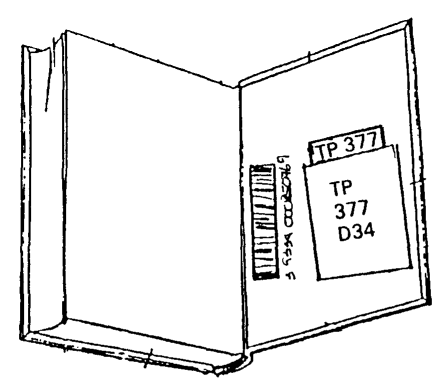

3. On pose ensuite le deuxième exemplaire du même code zébré (avec la mention «DUPL») sur la fiche.

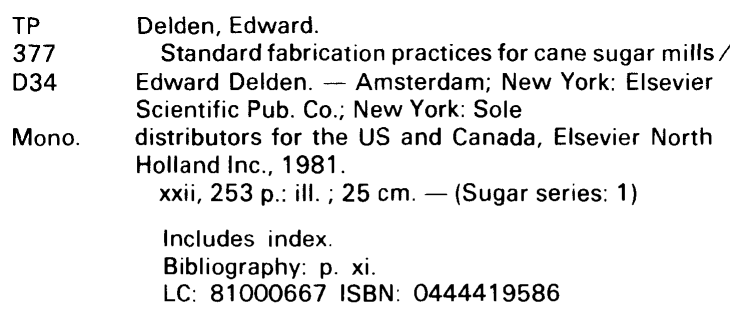

ÉTAT DE COLLECTION: Mono.

90 EPMZ

(Voir fiche suivante)

PRODUCED IN CANADA BY UTLAS Inc

OO01 830СT26 48138239 PRODUIT AU CANADA PAR UTLAS Inc. 


\section{Figure 1: Spécifications de conversion des notices}

Bibliographic conversion spécéfications Page: 03 Date: 30 apr. 1985

École Polytechnique, Montréal

22. If subfield \$c is absent in tag 090, input " 1 " in subfield \$c of the corresponding tag 930. If $090 \$ \mathrm{c}$ is present, copy its content as such into $930 \$ c$.

23. Copy the content of every $090 \$ d$ found into subfield $\$ \mathrm{k}$ of the corresponding tag 930 if the subfield starts with a string of 4 numeric characters, of which the first numeric character is = " $1 "$. If tag $090 \$ d$ starts otherwose than described, copy the content of $090 \$ d$ as such in subfield $930 \$ \mathrm{j}$ of the corresponding 930 .

24 . Copy the content of every $090 \$ r$ found into subfield $\$ i$ of the corresponding tag 930 .

25. For every tab 966 , create subfield $\$ m$ and its content according to specifications in List B attached.

26. List every occurrence of tag $090 \$ \mathrm{~m}$ and $090 \$ x$ found. Print also the corresponding record number (001) and call number (099\$a). Do not load $090 \$ m$ and $090 \$ x$.

\section{Do not load any tag 090, 092, or 265.}

Devant cette surcharge de travail, il a fallu procéder au plus urgent et suspendre temporairement certaines tâches de catalogage. Ainsi, dans la section des monographies, si la bibliothécaire n'avait que ses 600 notices à vérifier (conversion), les deux techniciennes en documentation devaient, en plus de leurs 600 notices chacune, régler les cas-problèmes amenés par les commis au zébrage. Il ne restait plus, pour le catalogage régulier, qu'un ratio d'une technicienne pour un bibliothécaire, alors qu'il avait été jusquelà de deux pour un.

On a donc cessé le catalogage rétrospectif des périodiques (voir schéma), ce qui a libéré complètement une technicienne et partiellement une autre qui devait continuer la mise à jour des titres courants et déjà catalogués. Cependant, une bibliothécaire est aussi devenue libre et lorsque la chargée du projet GLIS a eu besoin d'une assistante et qu'elle est venue chercher la technicienne nouvellement disponible, le problème du ratio bibliothécaire/technicienne s'est posé avec plus d'acuité: de 1 bibliothécaire pour 3,5 techniciennes qu'il était avant GLIS pour l'ensemble du service de catalogage, ce ratio était passé à une bibliothécaire pour 1,5 technicien en documentation. L'arrivée de GLIS avait donc considérablement augmenté la tâche, non seulement des
28. In tag 111,711 , or 811 (if any), change subfield code " $b$ " to " $n$ ".

29. In record range 48-000-001 to 48-025999 , if tag $300 \mathrm{Sa}$ is identical to one of the following codes, change the code to the corresponding equivalent:

$$
\begin{aligned}
& \text { LF becomes "env. } 10 \text { p." } \\
& \text { PF becomes "env. } 25 \text { p." } \\
& \text { BL becomes "env. } 75 \text { p." } \\
& \text { BK becomes "plus que } 100 \text { p. " }
\end{aligned}
$$

Please note that every equivalent is followed by a space, and the quotation marks are not parts of the equivalents (neither are they parts of any value or data quoted in this document).

30. In record range $48-000-001$ to $48-025$ 999 , change tag number 410 to 440 and set the first indicator of 440 to @ (blank).

31 . In record range 48-100-001 to 48-122999 , list every occurrence of tag 400,410 or 411 . Print also the corresponding record number (001) and call number $(099 \$ a)$. These tags must be loaded as such, however.

32. List every occurrence of tag 533 found. Print also the corresponding record number (001) and call number (099 \$a). This tag must be loaded as such, however.

bibliothécaires, mais surtout des techniciennes en documentation à cause de la technicité des nouvelles tâches.

\section{0 autres notices à corriger}

En septembre 1985, la pose des codes zébrés battait son plein et les bibliothécaires au catalogage avaient été partiellement affectés au traitement lui-même des documents tandis que les techniciennes en documentation réglaient les casproblèmes de zébrage. Pendant ce temps, la chargée de projet et son assistante travaillaient toujours à la conversion des 92000 notices de la bibliothèque pour assurer leur transfert de UTLAS à GEAC.

En septembre, le personnel du catalogage apprenait qu'il devait corriger 8000 notices qui comprenaient des erreurs assez importantes pour ne pas pouvoir être acceptées telles quelles dans le fichier GLIS. Ces erreurs étaient le plus souvent:

- des absences de zones MARC (par exemple, la zone de l'adresse bibliographique pour nos notices Codoc);

- des absences de sous-zones ou l'emploi de 
sous-zones invalides (par exemple, pour la zone du titre, l'emploi des «\$d» et «\$e» (anciennes notices) au lieu des $« \$ n »$ et $\ll \$ p »)$;

- des codes de zones fixes invalides ou absents (par exemple, l'emploi du code «h» périmé dans la zone de la source du catalogage au lieu du code «O»).

On a donc distribué ces 8000 notices au personnel qui a effectué les corrections pour chacune des zones MARC devant lesquelles GLIS avait mis une étoile. Certaines notices contenaient trois ou quatre étoiles!

\section{La chaîne de travail}

En novembre, il fallut repenser la chaîne de travail au catalogage car nous voyions arriver le jour où le nouveau module de catalogage serait fonctionnel. Et ce qui rendait les choses encore plus complexes, c'est que certaines composantes du nouveau module ne fonctionneraient pas dès le début. Ainsi l'interface qui nous permettrait de dériver des notices d'un fichier accessible sur UTLAS et ensuite de les transférer dans GLIS d'où nous pourrions les corriger et les adapter à nos besoins, n'était et n'est pas encore prête et le fichier des 15000 notices d'autorité est loin d'avoir vu le jour.

Le 2 décembre 1985 marque la mise en opération du nouveau module de catalogage GLIS et aussi le début de l'opération «jumelage» qui consiste à introduire dans le nouveau système les codes zébrés posés sur les fiches topo UTLAS.

La mise en opération du nouveau module de catalogage suppose de nombreuses réunions techniques, des séances de formation ou de rappel et aussi, un sentiment de perdre du temps à réorganiser chacun des aspects d'un travail qui est pourtant parfaitement connu. Pour diminuer un peu le nombre de réunions, le responsable du catalogage, en collaboration avec la chargée du projet GLIS, a rédigé plusieurs documents techniques expliquant les changements les plus importants dans le codage des bordereaux de catalogage.

\section{Opération jumelage}

Pendant cette phase de réorganisation des opérations les plus routinières de notre chaîne de travail et alors que les problèmes de codes zébrés ne sont pas encore tous résolus, il nous a fallu entamer l'opération jumelage qui doit être terminée à l'été de 1986 pour la mise à feu du module de prêt automatisé. Cette opération n'est qu'une simple lecture à l'aide d'un photostyle des 90000 étiquettes posées sur nos fiches topo UTLAS. Elle exige de lire le bon code zébré dans la bonne zone de format MARC. Par exemple, si une notice comprend douze codes zébrés, il faudra créer dans GLIS autant de fois la zone 966 (zone pour le prêt) qu'il y a de documents zébrés, lire les codes zébrés dans chaque zone 966 et, surtout, les identifier en conformité avec les fiches topo UTLAS.

La figure 2 représente une notice bibliographique telle qu'elle apparaît après avoir été jumelée. Pour arriver à ce résultat, le «jumeleur» a créé six zones 966 correspondant au nombre de codes zébrés. II a par la suite identifié ces zones de manière telle que chacune corresponde à une unité physique de l'état de collection:

\$d 1980
\$d 1981
\$d 1982
\$d 1983 se v. 1
\$d 1983 \$e v. 2
\$d 1984

C'est à ce moment seulement qu'il a été possible de «lire» avec le photostyle les codes zébrés posés sur la fiche. Mais attention! II faut lire le code zébré marqué «1981» sur la fiche dans la zone 966 marquée aussi «1981»! Sinon, c'est le fouillis au comptoir de prêt.

Chaque bibliothécaire et chaque technicienne du catalogage a convenu de consacrer deux heures par jour à la cause du jumelage. De plus, une technicienne en documentation du service des acquisitions apporte souvent son aide, ce qui porte l'équipe de travail à onze personnes disponibles. Ainsi, idéalement, près de 25000 jumelages par mois devraient être faits.

\section{Aspects humains}

Des techniciennes en documentation se sont vu confier différentes opérations nécessaires à l'implantation de GEAC à titre de personnes-ressources. Deux raisons ont justifié le choix de confier ces responsabilités à des techniciennes en documentation: les bibliothécaires travaillaient à temps partiel et ne connaissaient pas les pratiques locales et I'histoire du catalogage de la bibliothèque; on voulait enfin fournir l'occasion aux techniciennes en documentation de constater qu'elles font partie d'une équipe de travail et que l'on a confiance en elles. L'avenir confirmera sûrement que ce fut un facteur de motivation au travail.

Le personnel du service de catalogage n'est pas au bout de ses peines. On est tous au beau milieu de l'opération jumelage et l'adaptation à une nouvelle chaîne de travail se poursuit... même si elle est transitoire, car il manque encore plusieurs morceaux importants du casse-tête: interface, fichier de notices d'autorité. 
Figure 2:

Notice bibliographique après le jumelage

05 Geac Marc Data Entry System

Function: CHANGE

CMD: dsp TAG:

$\begin{array}{ll}001 & 1 \\ 008 & 1 \\ & 1 \\ 040 & 1 \\ 050 & 1 \\ 099 & 1 \\ 111 & 1 \\ 245 & 1 \\ 260 & 1 \\ 362 & 1 \\ 500 & 1 \\ & \\ 550 & 1 \\ & \\ 711 & 1 \\ 930 & 1 \\ 966 & 1 \\ 966 & 2 \\ 966 & 3 \\ 966 & 4 \\ 966 & 5 \\ 966 & 6\end{array}$

48136811.CG.

Saqmep\$bFRE

\$aTS 191.8157
Date: 86-05-22

Table: 01 as Source: M GRSN: 00035615

$\begin{array}{llll}\text { b } & 1 & \text { eng } & 0\end{array}$
830317 m19709999iluar

$4 \$ a T S 191.8 \$ b .157$

20 Salnternational Sympsium on Industrial Robots.

10 \$aProceedings of the... International Symposium on Industrial Robot s. -

01 \$aChicago: \$bllT Research Institute, \$c1970 — . — \$a \$blLL.; \$c29 cm. -

0 saist (1970) - . -

SaLa $10 \mathrm{e}, 1 \mathrm{a} 12 \mathrm{e}$ et la $14 \mathrm{e}$ conference comprennent aussi respectivement: 5 th, 6 th et 7th International Conference on Industrial Robot Technology.

1 \$aL'adresse bibliographique varie: 1980-1982, 1984 -, Bedford: IFS (Publications); 1983, Dearborn: Robotics International of SME.

20\$alnternational Conference on Industrial Robot Technology.

\$1GEN \$cex. 1:\$dTS 191.8157 \$k1980-1984.

\$c1\$1GEN \$mPRET \$sTS 191.8157 d1980 \$b39334000429603

\$c1\$1GEN \$mPRET \$sTS $191.8157 \$ d 1981$ \$b39334000429652

\$c1\$1GEN \$mPRET \$sTS $191.8157 \$ d 1982$ \$b39334000429702

\$c1\$1GEN \$mPRET \$sTS $191.8157 \$ d 1983 \mathrm{ev} .1$ \$b39334000429751

\$c1\$1GEN \$mPRET \$sTS 191.8157 \$d1983ev. 2 \$b39334000429769

\$c1\$1GEN \$mPRET \$sTS $191.8157 \$ d 1984$ \$b39334000434041
La transition de UTLAS à GEAC aura duré deux ans quand elle s'achèvera, à l'automne 1986. Les membres du personnel en ressortiront plus unis et auront réussi à former une véritable équipe de travail. Le point négatif de l'expérience est sans doute le stress ressenti par les équipes de travail et aussi une certaine frustration causée par l'obligation d'assurer un service de qualité aux usagers de la bibliothèque tout en assimilant et organisant une nouvelle façon de travailler. Et pour apprivoiser ces malaises, à défaut d'argent, d'espace et de ressources en personnel, nous avons choisi une approche plus humaine, axée sur la communication et l'humour.

Un mot encore sur les outils de contrôle employés pendant la durée de l'implantation de GLIS. Le responsable du traitement de la documentation diffusait régulièrement des rapports d'étape qui faisaient le point sur les différents travaux en cours. Le tableau 1 résume les activités de l'opération jumelage de décembre 1985 à avril 1986.

Peu importe l'ampleur et les raisons qui ont provoqué de tels changements au sein de la bibliothèque, il n'en demeure pas moins qu'ils ont suscité de nombreuses controverses, et les professionnels nouvellement engagés ont vite senti l'urgence d'établir un climat de confiance avec l'équipe qu'ils devaient superviser.

On ne peut passer sous silence l'appréhension des employés face à la surcharge de travail durant toute cette la période de l'implantation du nouveau système. Cet inconfort se manifestait par un sentiment d'insatisfaction ou d'infidélité face aux priorités quotidiennes. La chaîne de travail se trouvait en effet désorganisée ce qui obligea les responsables à intervenir fréquemment pour dissiper les craintes.

\section{De l'information à la collaboration}

Les superviseurs et responsables du projet eurent un rôle délicat à jouer tout au long des travaux de transition du système. Ils informèrent régulièrement l'équipe de travail de l'évolution des travaux et insistèrent sur l'importance de la qualité du rendement. Un bon réseau de communication fut instauré pour établir un climat de confiance réciproque. En effet, nous nous retrouvions dans une situation où l'employeur imposait à son personnel des changements technologiques en exigeant à la fois un taux de rendement supérieur sans ressources humaines additionnelles. S'ensui- 
Tableau 1 - Opération jumelage - Rapport d'étape

Décembre 1985 à avril 1986

\begin{tabular}{|c|c|c|c|c|c|c|c|}
\hline & Décembre & Janvier & Février & Mars & Avril & $\begin{array}{c}\text { Moyenne } \\
\text { ou total }\end{array}$ & $\begin{array}{l}\text { Moyenne ou } \\
\text { total pour LC }\end{array}$ \\
\hline $\begin{array}{l}\text { Total d'heures } \\
\text { consacrées au } \\
\text { jumelage }\end{array}$ & $\begin{array}{l}157 \text { hres } \\
\text { en } \\
2 \text { jours }\end{array}$ & $\begin{array}{l}177 \text { hres } \\
\text { en } \\
20 \text { jours }\end{array}$ & $\begin{array}{l}272 \text { hres } \\
\text { en } \\
20 \text { jours }\end{array}$ & $\begin{array}{l}252 \text { hres } \\
\text { en } \\
21 \text { jours }\end{array}$ & $\begin{array}{l}346 \text { hres } \\
\text { en } \\
20 \text { jours }\end{array}$ & $\begin{array}{c}1204 \text { hres } \\
\text { en } \\
93 \text { jours }\end{array}$ & $\begin{array}{l}748 \text { hres } \\
\text { en } \\
62 \text { jours }\end{array}$ \\
\hline $\begin{array}{l}\text { Heures par } \\
\text { jour par équipe }\end{array}$ & 13 hres & 9 hres & 14 hres & 12 hres & 17 hres & 13 hres & 12 hres \\
\hline $\begin{array}{l}\text { Heures par } \\
\text { jour par } \\
\text { personne }\end{array}$ & $1 \mathrm{~h} 12$ & 0 h48 & $1 \mathrm{~h} 13$ & $1 \mathrm{~h}$ & $1 \mathrm{~h} 20$ & $1 \mathrm{~h} 06$ & $1 \mathrm{~h} 12$ \\
\hline $\begin{array}{l}\text { Codes zébrés } \\
\text { lus }\end{array}$ & 7500 & 10587 & 17392 & 13933 & 17417 & 66829 & 44992 \\
\hline $\begin{array}{l}\text { Codes zébrés } \\
\text { lus par jour } \\
\text { par l'équipe }\end{array}$ & 625 & 529 & 870 & 664 & 871 & 719 & 726 \\
\hline $\begin{array}{l}\text { Codes zébrés } \\
\text { lus par jour } \\
\text { par personne }\end{array}$ & 57 & 48 & 73 & 55 & 67 & 60 & 63 \\
\hline $\begin{array}{l}\text { Jumelage à } \\
\text { l'heure par } \\
\text { personne }\end{array}$ & 48 & 60 & 64 & 55 & 50 & 55 & 60 \\
\hline $\begin{array}{l}\text { Nombre de } \\
\text { jumeleurs* }\end{array}$ & 11 & 11 & 12 & 12 & 13 & 12 & 11.5 \\
\hline
\end{tabular}

Pour le nombre de jumeleurs, nous avons compté les personnes et non leur disponibilité.

vent inévitablement des tensions, des plaintes et du stress dont il est important de limiter les effets néfastes.

La transition du système automatisé de CATSS à GEAC aura permis, il faut le souligner, d'établir un climat de coopération exceptionnel et de faire participer bibliothécaires et techniciens en documentation aux mêmes tâches lors de la conversion des notices (pose de codes zébrés, etc.). Cette attitude a permis d'apprécier davantage le produit fini. Les responsables s'efforcèrent d'accroître la motivation de leurs employés et de créer un climat de travail serein. Pour leur part, les techniciens en documentation furent valorisés par les travaux qu'on leur confiait. Quant aux responsables, ils devaient maintenir l'enthousiasme tout en tentant de prévoir les erreurs de parcours; ils assistaient à des rencontres d'information, concevaient et rédigeaient les rapports d'étape, ajustaient au besoin le calendrier des travaux, et transmettaient les informations pertinentes aux différentes équipes de travail.
Ganza Dakshnamurti ${ }^{1}$ mentionne que pour maintenir l'état de santé d'un projet, le responsable devrait être une personne enthousiaste, dynamique, flexible et, surtout, elle devrait faire preuve d'humour pour appaiser les inquiétudes et les tensions. Accepter les suggestions et les remarques des membres de l'équipe et être à leur écoute sont des qualités indispensables pour réussir un bon travail. Il faut donc savoir composer avec tous les facteurs humains et technologiques sil'on veut qu'un projet évolue sainement et atteigne sa pleine maturité. Ainsi, c'est avec philosophie et sagesse que la coordination du travail sera possible. Et tant qu'il y aura place au dialogue, l'exécution du travail sera mieux absorbée par le personnel et les priorités plus nuancées.

\section{André Maltais \\ Denise Mauffette \\ Services techniques \\ Bibliothèque \\ École Polytechnique \\ Montréal}

